Received: 2016.12 .15

Accepted: 2017.02 .08

Published: 2017.11.17

Authors' Contribution:
A Study Design
B Data Collection
C Statistical Analysis
D Data Interpretation
E Manuscript Preparation
F Literature Search
G Funds Collection

\title{
Ultrasound is Not Useful as a Screening Tool for Dengue Fever
}

\author{
Jitendra Parmar ${ }^{1 \mathrm{ABCDEF}}$, Chander Mohan ${ }^{2 \mathrm{ABCDEF}}$, G. Prem Kumar ${ }^{1 \mathrm{ACDF}}$, Maulik Vora ${ }^{3 \mathrm{ABCDEF}}$ \\ ${ }^{1}$ Department of Radiology, Dr. B. L. Kapur Hospital, New Delhi, India \\ 2 Department of Interventional Radiology, Dr. B. L. Kapur Hospital, New Delhi, India \\ ${ }^{3}$ Department of Radiology, Indira Gandhi Medical College, Shimla, Himachal Pradesh, India
}

Author's address: Maulik Vora, Department of Radiology, Indira Gandhi Medical College, Shimla, Himachal Pradesh, India, e-mail:dr_maulikvora@yahoo.com

Material/Methods:

\section{Summary}

Background: Dengue fever is a tropical disease that is transmitted by female Aedes Aegypti mosquitos. Early diagnosis is necessary to reduce the mortality and morbidity associated with the disease. A combination of clinical, laboratory, and sonography findings can be potentially helpful in making an early diagnosis of dengue fever. There is paucity of literature on the use of ultrasound for dengue fever screening; hence, the primary objective of the study was to evaluate the utility of ultrasound as a screening tool in dengue fever.

A total of 160 patients of suspected dengue fever were included in the study. They underwent ultrasound examinations in order to search for thickening of the gallbladder wall, pleural effusion, and ascites. On the basis of ultrasound findings, 65 cases were positive and 95 cases were negative for dengue fever. Serological tests were also used for diagnosing dengue fever, 93 cases were seropositive and 67 cases were seronegative. The ultrasonically diagnosed cases were compared with serologically diagnosed cases, and appropriate descriptive statistical analyses were applied.

Results: The ultrasound findings included gall bladder wall thickening in 66 patients (41.2\%). The sensitivity, specificity, and positive predictive value of ultrasound in diagnosing dengue fever were $58 \%, 84 \%$, and $83 \%$, respectively. The negative predictive value and accuracy were $59 \%$ and $68.8 \%$, respectively.

Conclusions: The present study suggests that increased gall bladder wall thickness, pleural effusion, ascites, hepatomegaly, and splenomegaly are highly suggestive of dengue fever in clinically suspected cases. However, ultrasound should not be used as a screening tool, as negative ultrasound findings cannot rule out dengue fever due to the low sensitivity of this examination.

MeSH Keywords:

Dengue • Mass Screening • Ultrasonography, Doppler, Color

Abbreviations:

DF - Dengue fever; WHO - World Health Organization; NS1 - non-structural 1 protein; SPSS - statistical package for the Social Sciences; PPV - positive predictive value; NPV - negative predictive value; $\mathbf{c m}$ - centimeter; $\mathrm{mm}$ - millimeter; $\mathbf{G B}$ - gall bladder

PDF file: $\quad$ http://www.polradiol.com/abstract/index/idArt/902861

\section{Background}

Dengue fever (DF) is a tropical disease. It is a mosquitoborne viral infection, usually transmitted by female Aedes Aegypti mosquitos. Common symptoms of DF include fever, headache, muscle and joint pains, and skin rash $[1,2]$. There exist four pathogenic serotypes of the dengue virus that belongs to the genus flavivirus (DEN-1, DEN-2, DEN-3 and DEN-4); however, all serotypes produce similar clinical manifestations [3,4]. 
In 2009, WHO reclassified dengue fever according to severity: DF without warning signs; DF with warning signs (abdominal pain, persistent vomiting, fluid accumulation, mucosal bleeding, lethargy, liver enlargement, increasing hematocrit with decreasing platelets); and severe DF (dengue with severe plasma leakage, severe bleeding or organ failure) [1]

Positive serology with clinical manifestation is the mainstay in the diagnosis of DF. However, serology takes approximately 7 days to give a positive result $[3,4]$, hence, the diagnosis of DF is often delayed [4]. Non-structural protein 1 (NS1) has been shown to be positive within four days of infection, with sensitivity of $71.4 \%$, but this test is expensive, not widely available, and becomes difficult in a larger population.

Early diagnosis is necessary to reduce the mortality and morbidity associated with this disease; hence, a combination of clinical, laboratory, and sonography findings can be potentially helpful in making an early diagnosis of dengue fever $[3,4]$. Based on the available literature, ultrasound has been used for detecting gall bladder wall thickening, ascites, pleural (right or bilateral) and pericardial effusion, hepatomegaly, splenomegaly, and pancreatic enlargement in patients with dengue fever. However, there is paucity of literature regarding the usefulness of the above-mentioned sonographic findings for screening of dengue fever.

\section{Material and Methods}

The primary aim was to study the utility of ultrasound as a screening tool in dengue fever.

\section{Ethical permission}

Dr. B. L. Kapur Memorial Hospital Ethics Committee and its Members and Scientific Research Committee and its Members have approved the study and provided ethical permission. All adult subjects and parents/guardians of underage participants have provided informed written consent for the study.

\section{Statistical analysis}

Type of study

Prospective observational study

\section{Statistical methods}

Statistical testing was conducted with the Statistical Package for the Social Sciences (SPSS), version 17.0. Sensitivity, specificity, PPV, and NPV were calculated to analyze the utility of ultrasound as a screening tool in dengue fever. For all statistical tests, $\mathrm{p}<0.05$ indicated a significant difference

\section{Method of evaluation}

The study was conducted in Dr. B. L. Kapur Memorial Hospital, New Delhi from July 2015 to December 2015. The study included 160 cases of suspected dengue fever, referred from the emergency and medicine department, who had clinical findings of dengue fever such as acute onset fever, frontal headache, myalgia, rashes, nausea, vomiting, and abdominal pain.

Abdominal ultrasound was performed with the GE LOGIO P6 machine, equipped with a 4C wide bandwidth (1.5 to 4.6 $\mathrm{MHz}$ ) convex probe and $11 \mathrm{~L}$ wide bandwidth (4 to $12 \mathrm{MHz}$ ) linear probe. A single radiologist with 10 years of experience in abdominal ultrasound, blinded to the study protocol, performed all ultrasound examinations. In all patients, abdominal ultrasound was performed after 4 hours of fasting for a better visualization of the gall bladder. Serial ultrasound examinations were done every other day.

Gall bladder wall thickening was measured by placing the calipers between the two layers of the anterior wall (thickened gall bladder wall: $\geq 3.0 \mathrm{~mm}$ )

Organomegaly, ascites/retroperitoneal fluid, and other additional findings were recorded. In adults, organomegaly was assessed objectively (hepatomegaly, $>15 \mathrm{~cm}$; splenomegaly, $>12 \mathrm{~cm}$; and pancreatic enlargement, $>30 \mathrm{~mm}$ at the pancreatic body region), while in pediatrics patient organomegaly was assessed objectively according to age and height.

Screening ultrasonography of the thorax was performed to look for pleural effusion. Both pleural spaces were evaluated through an intercostal approach. Thoracic scanning was done in either a sitting position or in supine posture.

\section{Investigations}

All cases had confirmed dengue fever by means of the dengue NSl antigen test or dengue IgM or IgG antibody test. Serological tests were performed on the same day as ultrasound. The dengue NSl antigen test is a lateral flow immunochromatography rapid test and is designed for in vitro qualitative detection of the dengue NSl antigen in human serum or plasma. The dengue IgG or IgM antibody test is a rapid immunochromatographic test that is used for qualitative detection of IgM and elevated titer of IgG antibodies to the dengue virus in human serum, plasma, and whole blood. A capture IgM and IgG ELISA assay using DENV/ JEV antigens and mAb reagents, provided by Venture Technologies, was used. These tests are used as screening tests for dengue fever, and they are performed in blood samples.

\section{Results}

A total of 160 suspected cases of dengue fever were included in the study. The study included 104 (65\%) males and 56 $(35 \%)$ females of various ages, ranging from 1 year to 77 years.

The clinical features included fever (100\%), rashes (5.6\%), headache $(42.5 \%)$, myalgia and body ache $(71.2 \%)$, nausea and vomiting $(68.1 \%)$, abdominal pain $(26.9 \%)$, retro-orbital pain $(2.5 \%)$, and other symptoms $(15.6 \%)$, such as loose motion, itching, joint pain, gum bleeding, black stool, seizures, giddiness, chest heaviness, breathlessness, drowsiness, and epistaxis (Table 1). 
Table 1. Clinical features.

\begin{tabular}{lrc}
\hline \multicolumn{1}{c}{ Symptoms } & \multicolumn{2}{c}{ Total $(\mathbf{n}=\mathbf{1 6 0})$} \\
\hline Fever & 160 & $(100 \%)$ \\
\hline Rashes & 11 & $(5.6 \%)$ \\
\hline Headache & 70 & $(42.5 \%)$ \\
\hline Myalgia/body ache & 112 & $(71.2 \%)$ \\
\hline Nausea/vomiting & 108 & $(68.1 \%)$ \\
\hline Abdominal pain & 42 & $(26.9 \%)$ \\
\hline Retro-orbital pain & 5 & $(2.5 \%)$ \\
\hline Others* & 25 & $(15.6 \%)$ \\
\hline
\end{tabular}

* Others: loose motion, itching, joint pain, gum bleeding, black stool, seizures, giddiness, chest heaviness, breathlessness, drowsiness, epistaxis.

The patients underwent ultrasound examinations before a serological confirmation of the disease. The ultrasound finding included gall bladder wall thickening in 66 patients $(41.2 \%)$, hepatomegaly in 30 patients $(18.7 \%)$, splenomegaly in 49 patients (30.6\%), ascites/retroperitoneal fluid in 49 patients (30.6\%), right-sided pleural effusion in 34 cases $(21.2 \%)$, left-sided pleural effusion in 18 patients (11.2\%), and bilateral pleural effusion in 18 patients (11.2\%).

Based on the ultrasound findings, 65 cases were considered positive for dengue fever and 95 cases were considered negative for dengue fever. These cases were correlated with serological tests. Out of total 160 cases, 93 cases were serologically positive for dengue fever and 67 cases were negative for dengue fever.

The ultrasound findings in serologically positive cases of dengue fever included gall bladder wall thickening in 54 cases $(58.0 \%)$, hepatomegaly in 21 cases $(22.5 \%)$, splenomegaly in 30 cases (32.2), ascites/retroperitoneal fluid in 40 cases $(43.0 \%)$, right-sided pleural effusion in 34 cases (36.5\%), left-sided pleural effusion in 18 cases (19.3\%), and bilateral pleural effusion in 18 cases (19.3\%). Figure 1 shows representative images of the various ultrasound findings in dengue fever.

These ultrasonically diagnosed cases were compared with serologically diagnosed cases of dengue fever for estimating sensitivity, specificity, positive predictive value, and negative predictive value of ultrasound findings in diagnosing dengue fever, with serology as the reference standard. Based on the ultrasound findings, 54 cases were true positive, 56 cases were true negative, 11 cases were false positive, and 39 cases were false negative (ultrasound findings of representative cases from each category are shown in Figures 2-5, respectively). Based on these findings, sensitivity, specificity, positive predictive value, and negative value were $58 \%, 84 \%, 83 \%$, and $59 \%$, respectively. The accuracy of ultrasound for diagnosing dengue fever was $68.8 \%$ (Table 2).

\section{Discussion}

Dengue fever is an acute febrile viral disease caused by the dengue virus. The disease is transmitted to people through an infected Aedes Aegypti mosquito, and it has an incubation period of 3-14 days. The incidence of dengue fever has increased manifold over the last 4-5 decades. Up to 50-100 million infections are now estimated to occur annually in over 100 endemic countries, putting almost half of the world's population at risk [2]. It increases the morbidity in middle-aged patients, leading to low productivity. The diagnosis of dengue fever is often delayed due to the time necessary for obtaining serological results. Early diagnosis is necessary to reduce the mortality and morbidity associated with this disease; hence, a combination of clinical, laboratory, and sonography findings can be potentially helpful in making an early diagnosis of dengue fever [3,4].

This study included 160 suspected cases of dengue fever, including 93 serologically confirmed and 67 serologically negative dengue fever patients. The age ranged between 1-77 years, 104 cases were male (65\%) and 56 (35\%) cases female. Among the 93 positive dengue fever cases, 60 $(64.5 \%)$ patients were male and $33(35.4 \%)$ were female. In the present study, the ultrasound findings included gall bladder wall thickening in 66 patients $(41.2 \%)$, hepatomegaly in 30 patients (18.7\%), splenomegaly in 49 patients $(30.6 \%)$, ascites in 49 patients $(30.6 \%)$, right-sided pleural effusion in 34 cases $(21.2 \%)$, left-sided pleural effusion in 18 patients (11.2\%), and bilateral pleural effusion in 18 patients (11.2\%). Out of these ultrasound findings, 54 cases of gall bladder wall thickening, 21 cases of hepatomegaly, 30 cases of splenomegaly, 40 cases of ascites, 34 cases of right-sided pleural effusion, 18 cases of left-sided pleural effusion and 18 cases of bilateral pleural effusion were serologically positive for dengue fever.

Gall bladder wall thickening was the most common finding in the present study ( $58 \%$ of cases), similarly to the studies by Quiroz-Moreno et al. (86\%) of the [5] and by Sai et al. (56\%) [6].

Gall bladder wall thickening was reported to be significantly associated with the severity of clinical manifestations of dengue virus infections. The present study showed gall bladder wall thickening in $96.5 \%$ of patients with severe dengue fever, in $57.6 \%$ of patients with dengue fever with warning signs, and in $28.9 \%$ of patients with dengue fever without warning signs. These findings were consistent with findings of Mehdi et al. who observed gall bladder wall thickening in all patients with severe dengue fever and in $86.6 \%$ of patients with mild dengue fever ${ }^{[7]}$. Setiawan et al. observed gall bladder wall thickening in $95 \%$ of patients with severe dengue fever and in $32 \%$ of patients with mild dengue fever [8]. The mean gall bladder wall thickening was $3.32 \mathrm{~mm}$ in dengue fever without warning signs, $4.95 \mathrm{~mm}$ in dengue fever with warning signs, and $8.80 \mathrm{~mm}$ in severe dengue fever. This observation was consistent with the findings reported by Colbert et al. who documented mean gall bladder wall thickening of $3.31 \mathrm{~mm}$ in mild dengue fever and $6.21 \mathrm{~mm}$ in severe dengue fever [9]. 


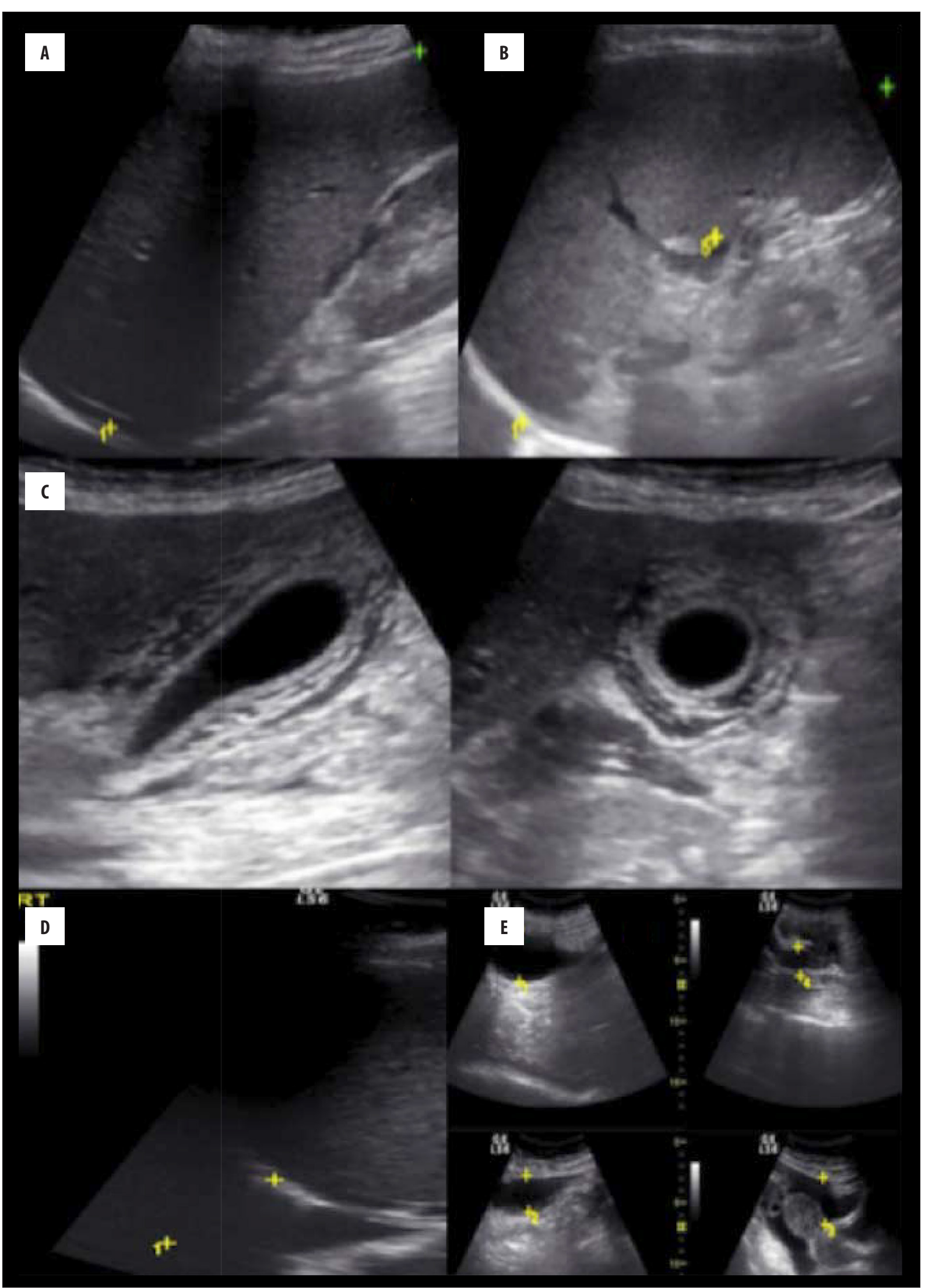

Figure 1. Ultrasound findings in dengue fever. Hepatomegaly (A), splenomegaly (B), diffuse gall bladder wall thickening of $7.3 \mathrm{~mm}$ with pericholecystic edema and minimal pericholecystic fluid (C), right-sided pleural effusion (D) and moderate ascites (E). 


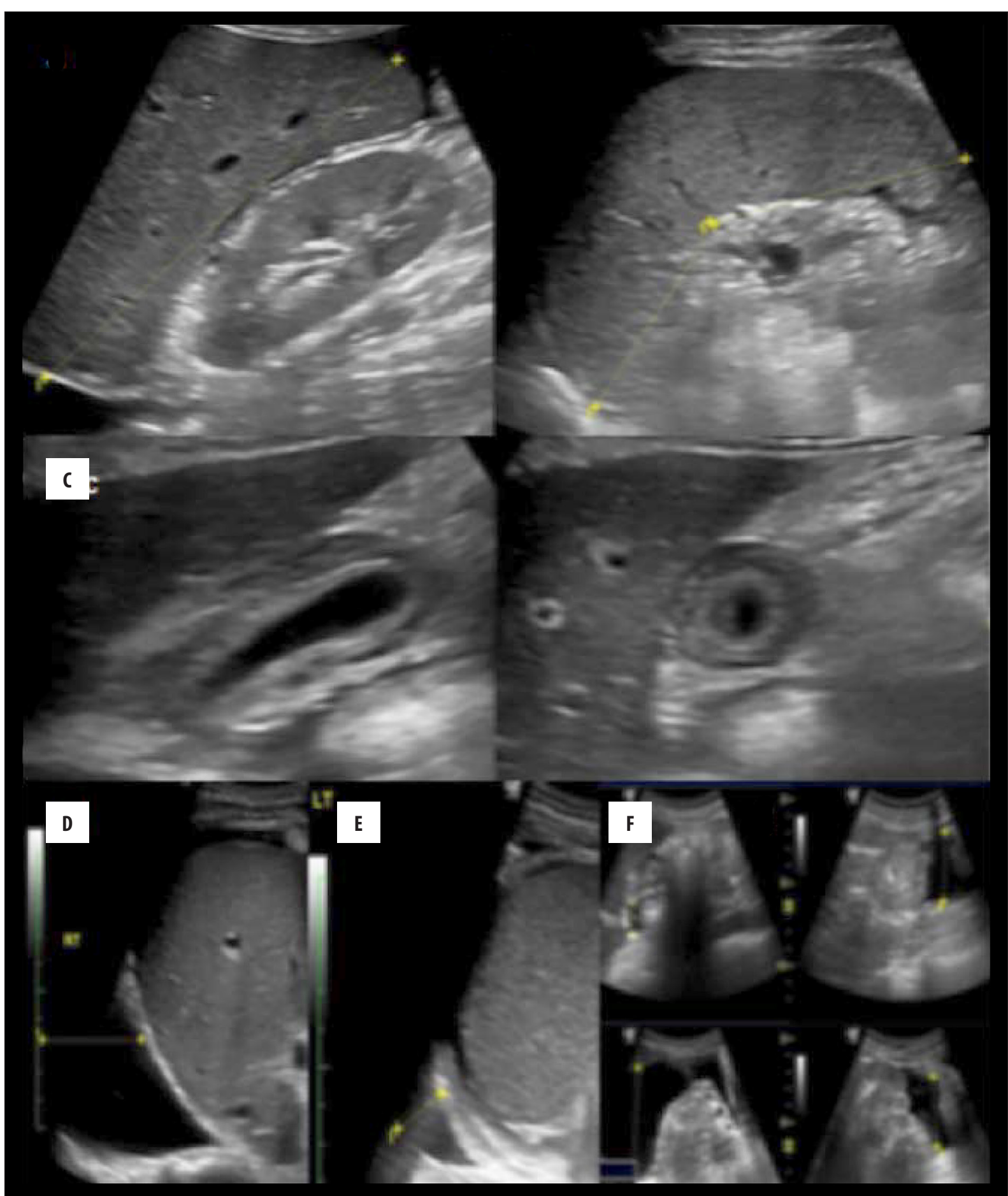

Figure 2. True positive case of dengue fever - ultrasound findings. A 23-year-old male patient presented with a history of fever for 4 days with nausea, vomiting, and abdominal pain. Ultrasound shows normal sized liver (A), splenomegaly (B), diffuse gall bladder wall thickening $(9.7 \mathrm{~mm}$ ) with pericholecystic edema (C), moderate right-sided pleural effusion (D), mild left-sided pleural effusion (E) and severe ascites (F) on day 4 of the fever. The patient was sonographically positive for dengue fever, which was confirmed by laboratory investigations. 


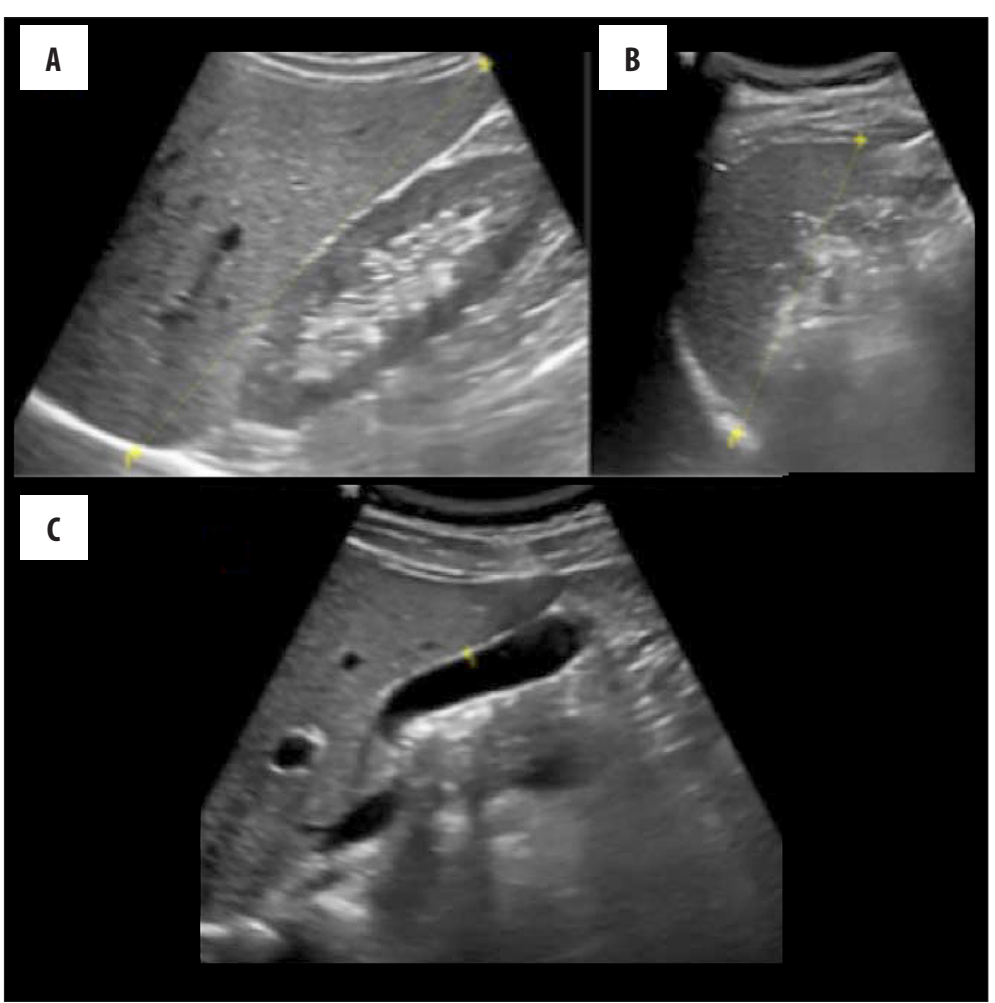

Figure 3. True negative case - ultrasound findings. A 22-year-old female patient came with a history of fever, nausea, vomiting, myalgia, body ache, and abdominal pain for 4 days. Ultrasound shows normal liver (A), spleen (B) and normal gall bladder wall thickness on day 4 of the fever. The patient was sonographically negative for dengue fever, which was confirmed by negative laboratory investigations.

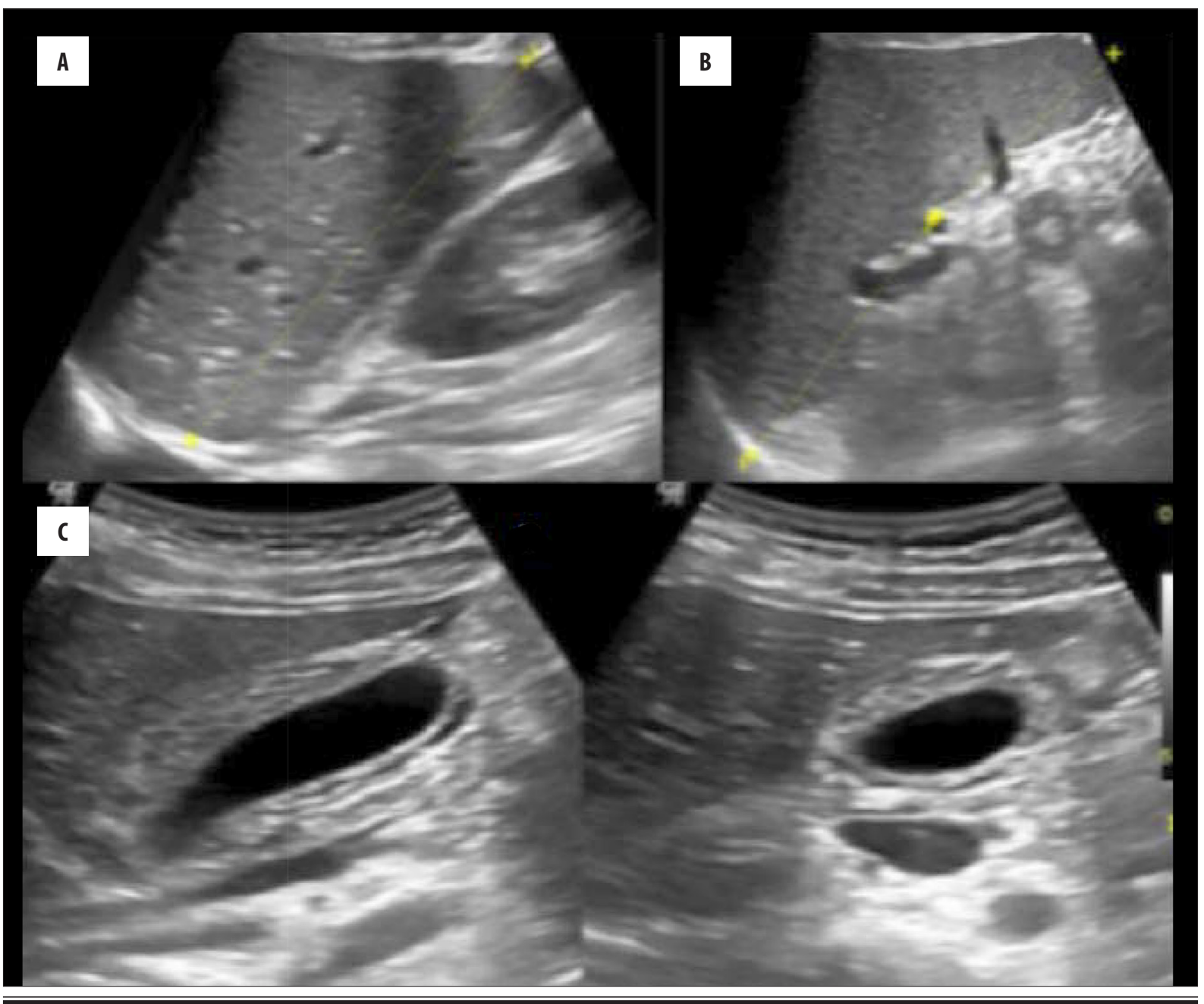




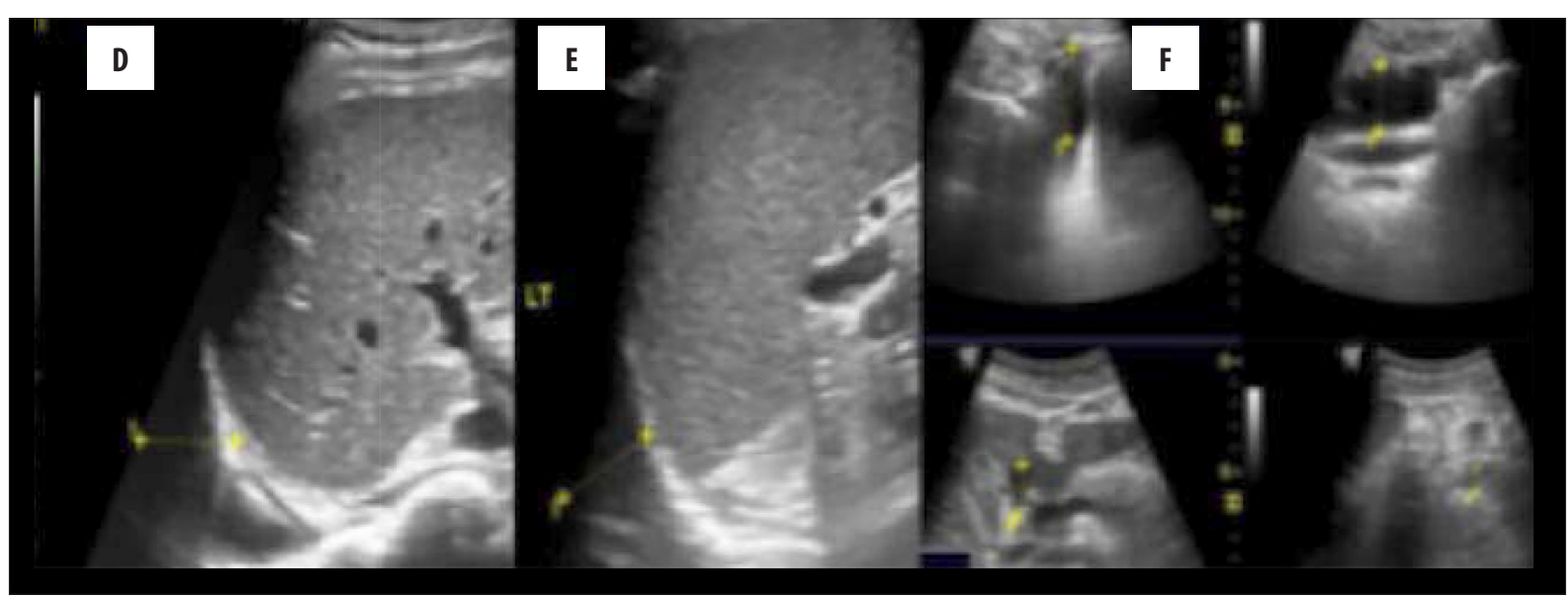

Figure 4. False positive case - ultrasound findings. A 23-year-old female patient presented with a history of fever for 3 days, myalgia, body ache, nausea, and vomiting. Ultrasound shows mild hepatosplenomegaly $(\mathbf{A}, \mathbf{B})$, diffuse gall bladder wall thickening of $6.2 \mathrm{~mm}$ with mild pericholecystic edema $(\mathbf{C})$, mild bilateral pleural effusion $(\mathbf{D}, \mathbf{E})$ and mild ascites $(\mathbf{F})$ on day 3 of the fever. The patient was positive for dengue fever on ultrasound, but on serology turned out to be negative.

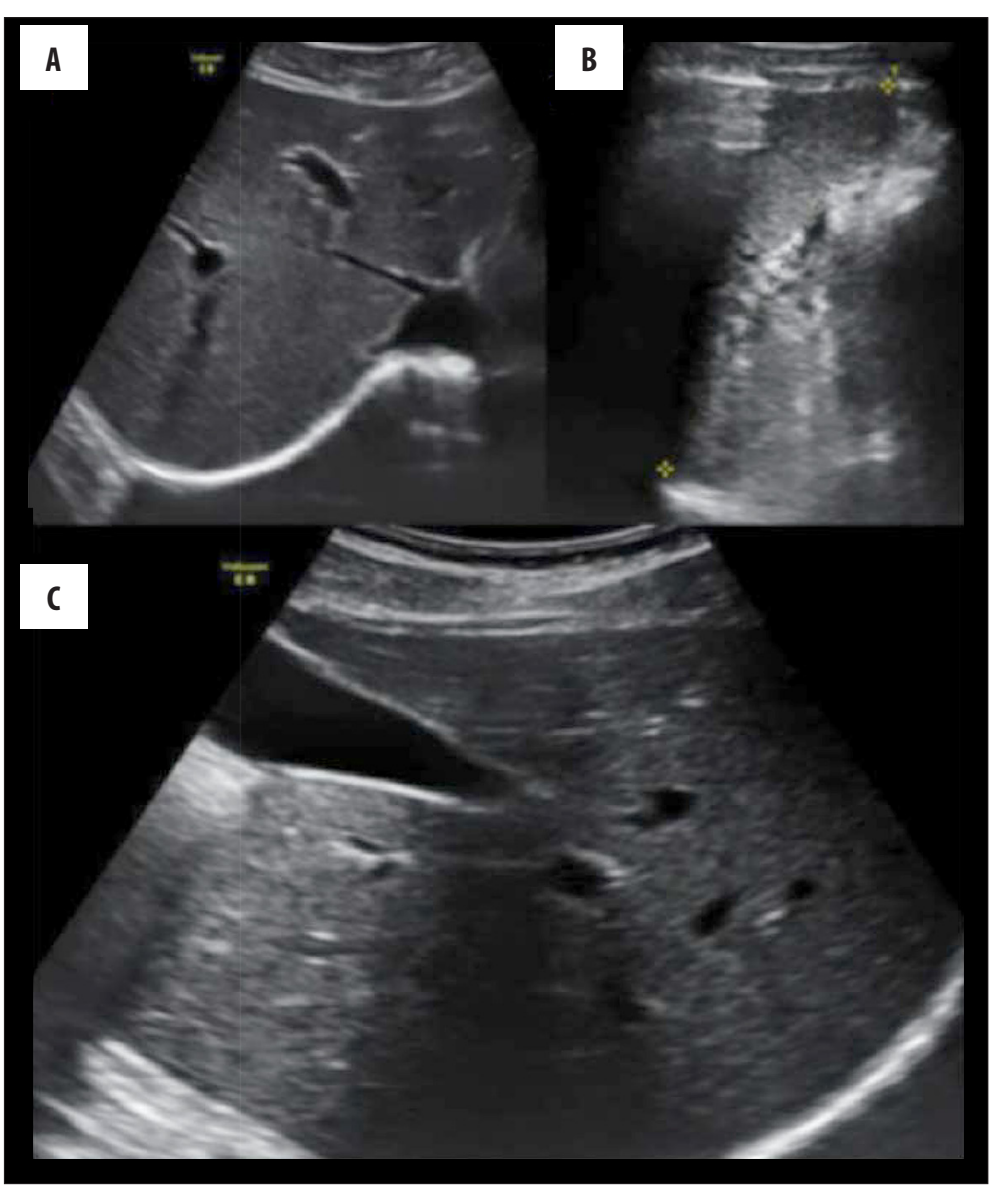

Figure 5. False negative case - ultrasound findings. A 28-year-old male presented with a history of fever, nausea, vomiting, and abdominal pain for 3 days. Ultrasound shows normal sized liver (A) and spleen (B) and normal wall thickness of the gall bladder $(\mathbf{C})$ on day 3 . The patient was sonographically negative for dengue fever, but serology was positive for dengue fever.

A prospective study performed by Keng-Liang wu et al. in 2004 that aimed to identify early sonographic features of dengue fever showed that sonographic features of DF include thickened gall bladder wall, ascites, splenomegaly, and pleural effusion (either right-sided or bilateral) [10]. The authors concluded that in epidemic areas, abdominal sonography could be used as the first-line imaging modality in suspected cases of dengue fever for detecting early signs of the disease. The findings of the present study are consistent with that study. The ultrasound can be used as the first-line imaging modality in suspected cases of dengue fever for early diagnosis of severe dengue fever. However, it cannot be used to rule out dengue fever, as the sensitivity of ultrasound is very low, as indicated by our results.

Santhosh et al. conducted a similar study for early diagnosis and assessment of dengue fever in 96 positive cases of dengue fever. The authors concluded that the sonographic 
Table 2. Sensitivity, specificity, positive predictive value, and negative predictive value.

\begin{tabular}{lc}
\hline & $\begin{array}{c}\text { Point estimates and 95\% } \\
\text { confident intervals }\end{array}$ \\
\hline True prevalence & $0.58(0.50,0.66)$ \\
\hline Sensitivity & $0.58(0.47,0.68)$ \\
\hline Specificity & $0.84(0.73,0.92)$ \\
\hline Positive predictive value & $0.83(0.72,0.91)$ \\
\hline Negative predictive value & $0.59(0.48,0.69)$ \\
\hline Positive likelihood ratio & $3.54(2.01,6.24)$ \\
\hline Negative likelihood ratio & $0.50(0.39,0.65)$ \\
\hline
\end{tabular}

features of thickened gall bladder wall, pleural effusion, ascites, hepatomegaly, and splenomegaly strongly suggested the diagnosis of dengue fever [11]. The ultrasound findings in their study are consistent with the results of the present study. However, their study did not estimate the sensitivity of ultrasound for the diagnosis of dengue fever. The findings of the present study suggest that ultrasound cannot be used as a screening tool for the diagnosis of dengue fever, as its sensitivity and negative predictive value for the diagnosis of dengue fever are very low, 58\% and 59\% respectively; however, specificity and positive predictive value are high, $84 \%$ and $83 \%$, respectively; with accuracy of $68.8 \%$. Our study has few limitations, like subjective variability in searching for findings on ultrasound, technical difficulties in performing laboratory tests for Dengue confirmation etc.
A "honeycomb" pattern in the thickened gall bladder wall on ultrasound specifically suggests the diagnosis of DHF. In an appropriate clinical context, when USG shows a thickened gall bladder wall with a honeycomb pattern, ascites, splenomegaly, and pleural effusion in a febrile patient in a DF endemic area, DHF should be considered until proven otherwise [12].

Sonography is a good tool for early diagnosis in clinically suspected cases of dengue fever, before serological studies are known, especially when dengue NSl is not available [13]. One should consider dengue fever when sonography shows a thickened gallbladder wall, ascites, splenomegaly, and pleural effusion in an acute febrile patient with thrombocytopenia in an area of recurrent dengue fever epidemics. The chest and abdomen ultrasound could be used as an additional diagnostic modality for early diagnosis of suspected dengue fever in endemic areas [14].

\section{Conclusions}

The present study suggests that such ultrasound findings as increased gall bladder wall thickness, pleural effusion, ascites, hepatomegaly, and splenomegaly in clinically suspected dengue fever cases, especially in the endemic areas, are highly suggestive of dengue fever. However, as the sensitivity and negative predictive value of ultrasound are very low, ultrasound should not be used as a screening tool, and negative ultrasound cannot rule out dengue fever. In view of the high specificity and positive predictive value, ultrasound may be used for early diagnosis of dengue fever, if serological diagnostic modalities, such as the NSl antigen test, are not available.

\section{References:}

1. Hadinegoro SRS. The revised WHO dengue case classification: does the system need to be modified? Paediatr Int Child Health 2012;32(supl): 33-38

2. Daumerie D, Savioli L, Crompton DWT, Peters P. Working to overcome the global impact of neglected tropical diseases: First WHO report on neglected tropical diseases. World Health Organization, 2010

3. World Health Organization, Research SP for, Diseases T in T, Diseases WHOD of C of NT, Epidemic WHO, Alert P. Dengue: guidelines for diagnosis, treatment, prevention and control. World Health Organization, 2009

4. Gubler DJ: Dengue and dengue hemorrhagic fever. Clin Microbiol Rev, 1998; 11(3): 480-96

5. Quiroz-Moreno R, Mendez GF, Ovando-Rivera KM: [Clinical utility of ultrasound in the identification of dengue hemorrhagic fever]. Rev Medica Inst Mex Seguro Soc, 2005; 44(3): 243-48

6. Sai PV, Dev B, Krishnan R: Role of ultrasound in dengue fever. Br J Radiol, 2005; 78(929): 416-18

7. Mehdi SA, Mahais AH, Bhukhari H, Aslam S: Gray scale transabdomino-thoracic ultrasonography in evaluation of dengue hemorrhagic fever. In: APMC. 2012; 32-36
8. Setiawan MW, Samsi TK, Pool TN et al: Gallbladder wall thickening in dengue hemorrhagic fever: An ultrasonographic study. J Clin Ultrasound, 1995; 23(6): 357-62

9. Colbert JA, Gordon A, Roxelin R et al: Ultrasound measurement of gallbladder wall thickening as a diagnostic test and prognostic indicator for severe dengue in pediatric patients. Pediatr Infect Dis J, 2007; 26(9): 850-52

10. Wu KL, Changchien CS, Kuo CH et al: Early abdominal sonographic findings in patients with dengue fever. J Clin Ultrasound, 2004; 32(8): $386-88$

11. Santhosh VR, Patil PG, Srinath MG et al: Sonography in the diagnosis and assessment of Dengue fever. J Clin Imaging Sci, 2014; 4: 14

12. Sachar S, Goyal S, Sachar S: Role of ultrasonography ("Honeycomb Sign") in early detection of Dengue hemorrhagic fever. Arch Clin Exp Surg, 2013; 2(1): 38-42

13. Chatterjee R, Mysore A, Ahya K et al: Utility of sonography in clinically suspected Dengue. Pediatr Infect Dis, 2012; 4(3): 107-11

14. Mia MW, Nurullah AM, Hossain A, Haque MM: Clinical and sonographic evaluation of dengue fever in Bangladesh: A study of 100 cases. Dinajpur Med Col J, 2010; 3(1): 29-34 